\title{
Measuring Non-geometric Soft Organ in Human Body, Liver Volume using Algorithms Bank Template
}

\author{
Amir Mohamed Elamir \\ IVI.UKM \\ Bangi, Malaysia
}

\author{
Riza Sulaiman \\ IVI.UKM \\ Bangi, Malaysia
}

\author{
Khalda F. Ali \\ College of Computer Science \\ PNU
}

\begin{abstract}
Many of the patients in liver functions need to donors that organ. Measurement of the liver needs optimal cute, accurate and faster. The measuring non-geometric soft organs in the human body using algorithms bank method to obtain the measuring liver volume with high accuracy, reducing the error rate and more fasting. In the realization of the objectives of this paper, developing a platform for measuring the liver volume with high accuracy and reduce the error rate, designing and develop the algorithm or more to calculate the liver volume with high accuracy and reduce the error rate, designing and developing a computer-aided measurement of liver volume in hepatic CT and MRI using computer graphics and visualization. The research method of this paper divided into four phases, phase one is image source, which is used to convert image source from CT and MRI to DICOM file. Phase two software application and development, which is used to remove image noise, enhance the liver boundary and liver image segmentation. Phase three template prototype application and measurement, which is used to create a template, compare the source image DICOM file with the template and restore an image as a template. Phase four compare the liver volume measurement with Gold standard reading, which is used to determine accuracy and error rate. The framework represents in three layers, technique layer, image layer and application layer. The research focusing on the application layer, which is divided into four stages represent in removing the image noise (internal and external) by Anisotropic diffusion filter and Median filter. Enhancing the liver boundary by A scale-specific gradient magnitude filter and Gaussian filter. Segmentation by hybrid based framework method using three algorithms, fast marching, Geodesic active contour, and level set. To make sure the fading of mistake and access to the value is zero, use one of the Artificial Intelligence techniques, which is the Artificial Neural Network. The template is a method to measure liver volume, which uses the template to measure the volume of the liver automatically instead of using the manual since the manual used the Gold standard method.
\end{abstract}

\section{General Term}

The general term of this paper focusing on computer graphics and visualization, which are used to measuring non- geometric shape (soft organ) in the human body such as liver volume.

\section{Keywords}

Computer graphics, remove noise, enhancement boundary, Artificial Neural Network, the gold standard, segmentation.

\section{INTRODUCTION}

Measuring liver volume passed in multiple phase's time, each of these phases has been the achievement. Measuring liver volume needs to plan an integrated method to be implemented to solve the non-geometric liver volume, taking into consideration building template, which is used to be the liver volume measuring with more accuracy, less rate error.

The present study was intended to develop the liver volume measurement method for better accuracy and time efficiency in liver segmentation $[1,3]$. The accuracy and time efficiency of the proposed algorithm method for liver segmentation were compared with those of the $2 \mathrm{D}$ and $3 \mathrm{D}$ region growing method implemented. Computer graphics and visualization liver extraction from hepatic MRI and CT images are challenges because the liver often abuts many organs of a similar density [2]. The one objective of the purposes of this paper was to develop a computer-aided measurement of liver volume in hepatic MRI and CT.

\section{METHOD}

The method conducts how to achieve the objectives. The objectives represent in four phases. Each phase describes separately. These phases represent the first phase is an image; the get image from the main source (ultrasonography), these sources are MRI or CT. the second phase represents in image manipulation; remove the noise, enhance the image and segmentation [3, 5]. The third phase is template prototype; which create a template, compare an image with the template and restore an image as a template, the fourth phase measuring the liver volume by the template. Figure 1 shows the method phases [4].

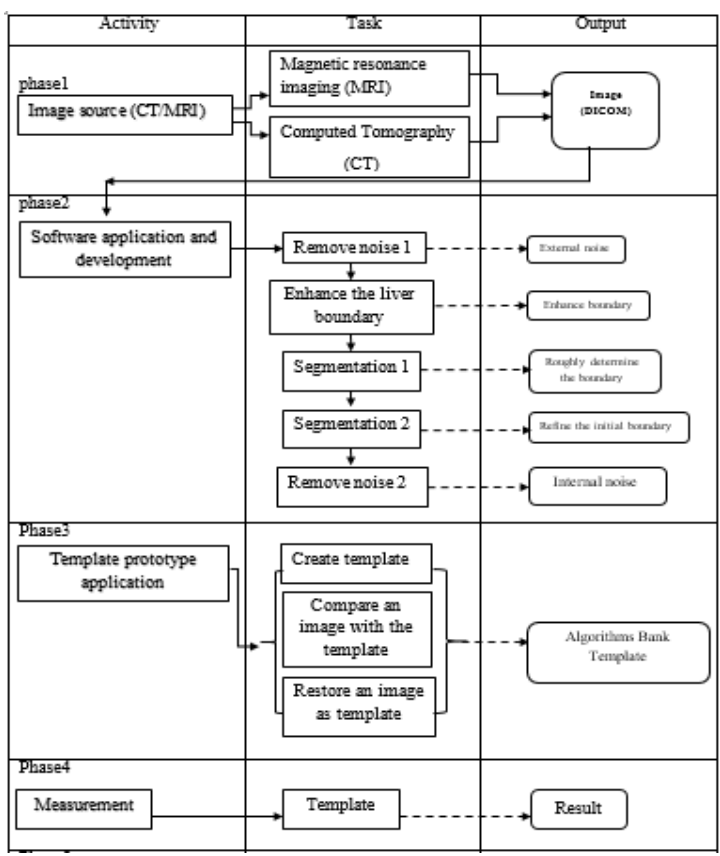

Fig 1. Method Phases 


\subsection{Phase1}

Magnetic Resonance Imaging(MRI) and Computed Tomography (CT) are sources of the technique used in the field of medical imaging, there are many other sources that are used in the field of medical imaging [6]. These two resources MRI and CT, the image can be converted to Digital Imaging and Communications in Medicine (DICOM), then the image has taken (DICOM EXTENSION), the Dicom image can be analyzed and manipulated in the second phase, which is the software application and development.

\subsection{Phase2}

This phase represents the one of main part focusing in this paper. The software application and development phase include four stages are as follows uses the algorithms bank. Remove the external noise; which uses the Anisotropic diffusion filter. The Anisotropic diffusion filter removes the Portal vein and liver border. Enhance liver boundary, which uses two filter algorithms to enhance the liver boundary, those filters are A scale specific gradient magnitude filter and Gaussian filter. Segmentation, which includes two parts, part one uses Fast marching algorithm, in this part gives the roughly determine liver boundaries, part two uses Geodesic Active Contour algorithm and Level-set algorithm, in this part gives the refine initial boundary[9]. Remove internal noise, which uses impulse noise to remove impulse noise. Figure 2 shows the phase 2 steps such as algorithms, stages, and the effects.

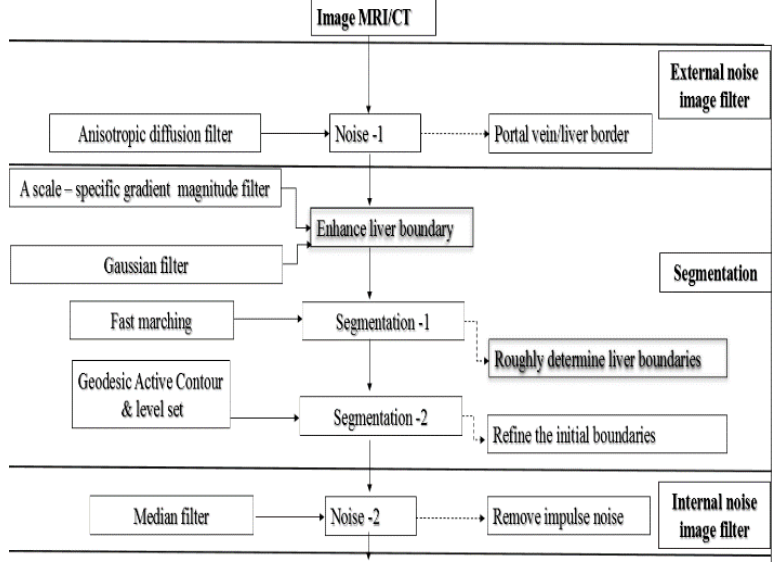

Fig 2. Bank Algorithm's Stages and the Effects

\subsection{Phase3}

In this phase represents the configuration templates that are measured by the image taken from MRI and CT. This phase contains three stages; create measurement template, compare the image with the measurement template and storing image as a template[7]. The measurement template represents the manual measurement after converted to auto measurement by this stage, this stage follows by next stage which is comparing the access new image with the suitable template to obtain the measurement with more accuracy and no error rate. The third stage stores the image as a template if the last image has no template before and use it as a template. Figure 3 shows the template prototype application [8].

\subsection{Phase4}

In this phase is matched results extract through the prototype application with the results of manual (Gold standard), "A gold standard is a method that is presumed to be correct for determining the presence of an abnormality or the parameter being estimated" $[2,5]$. The resulting from prototype compares with Gold standard, where identify the accuracy rate and error rate.

\section{ALGORITHMS BANK MATHEMATICAL FRAMEWORK}

Algorithms bank mathematical model framework is the summarized for each of the seven algorithms used in this paper, where the mathematical framework shows the activity algorithm's name and formula when implemented across this framework. The following table shows that table Seven Algorithms formula and activities.

Table 1. Activities, Algorithms, and formula

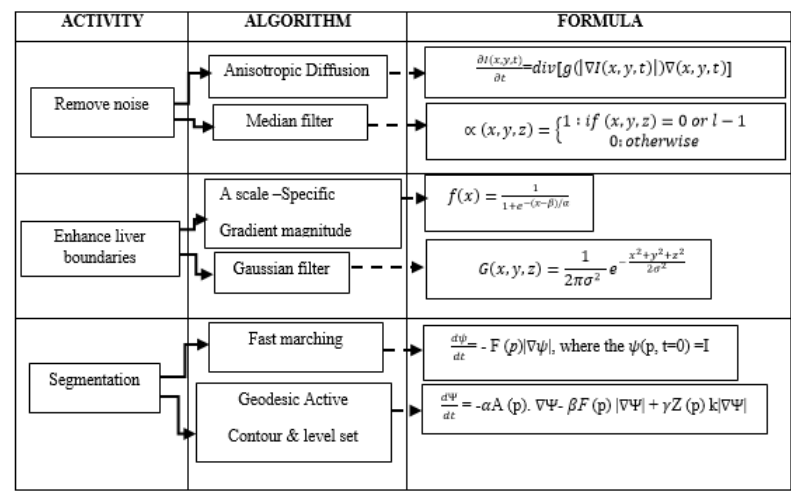

\section{PROTOTYPE APPLICATION}

Regarding the previous method and mathematical framework, it was created software program doing the method phases. The software application can be used to perform four functions, that functions remove the DICOM image noise, enhancement DICM image boundary, DICOM image segmentation and liver measurement. The user can select one modality DICOM image either CT or MRI, then can be manipulated by the previous functions. Figure 3 displays the application main screen.

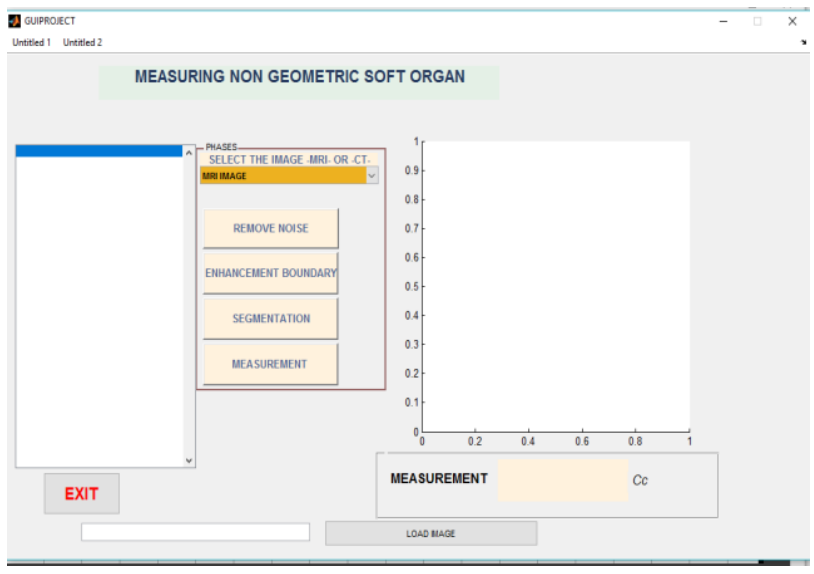

Fig 3. Main Screen Prototype Application

The main screen prototype application allows the user to select the image to be processed, where there are two options either CT image or MRI image in Dicom extension. After determining the image, the application starting with the first phase, this phase removes the noise, in this phase uses two algorithms, median filter algorithm, and Anisotropic diffusion algorithm. Figure 4 displays the two algorithms effect. 


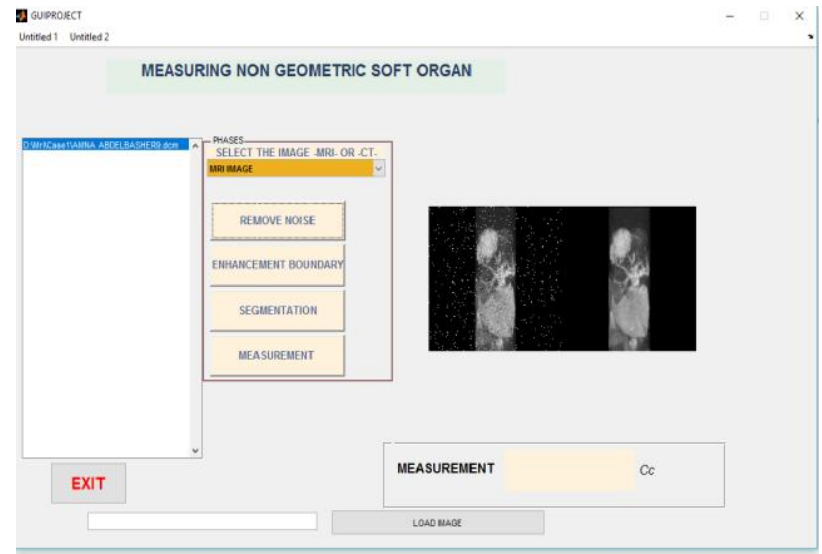

Fi4. Remove the noise by median filter algorithm and anisotropic diffusion, image $A$ before removing the noise, image $B$ after removing the noise

Scale-specific gradient magnitude filter algorithm and Gaussian filter algorithm use in enhancement boundary in the second phase. Figure 5 displays the enhancement boundary by are A scale specific gradient magnitude filter and Gaussian filter.

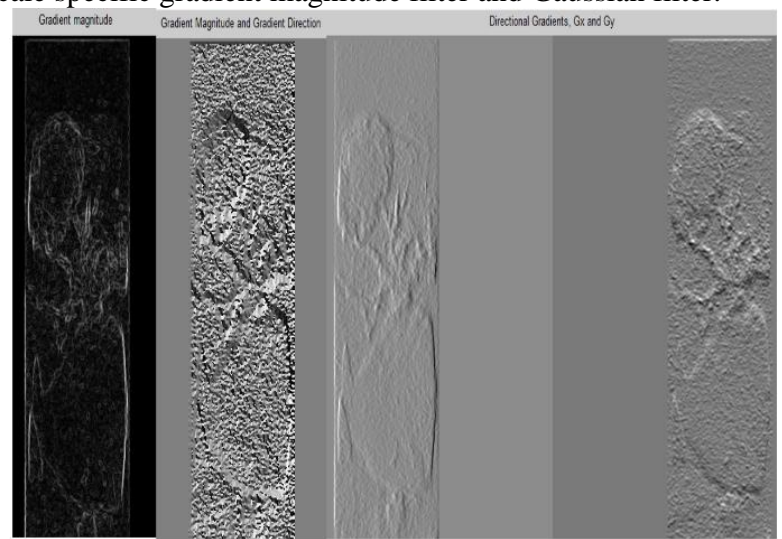

Fig5. A scale - specific gradient magnitude filter and Gaussian filter

Fast marching algorithm, Geodesic Active Contour algorithm, and level set algorithm to use in segmentation phase. Figure 6 displays the segmentation phase.

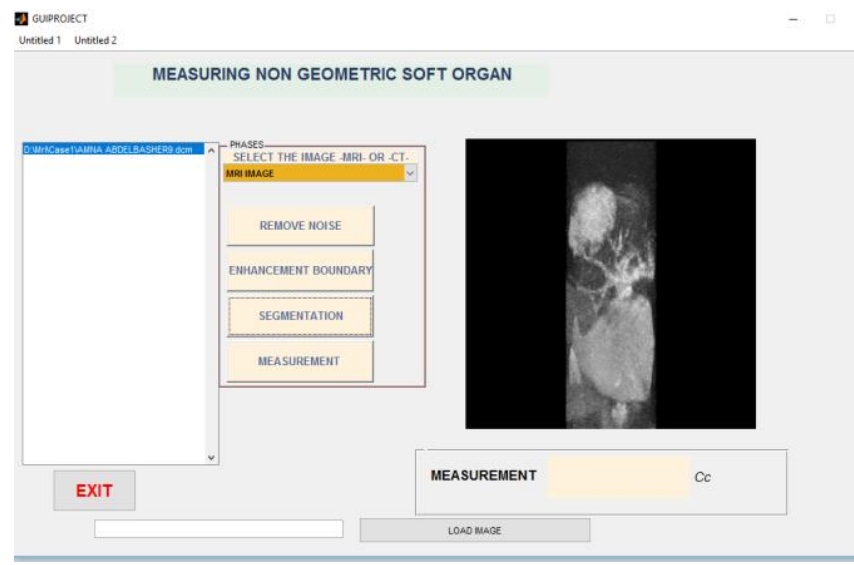

Fig 6. Displays the segmentation phase.

Measurement phase determines the measurement liver volume according to the previous phases, the prototype application determines the liver area and calculates, then compare the result with Gold standard using the artificial neural network, following histogram shows black color for the liver area and identified the liver measurement. Figure 7 displays the liver area.

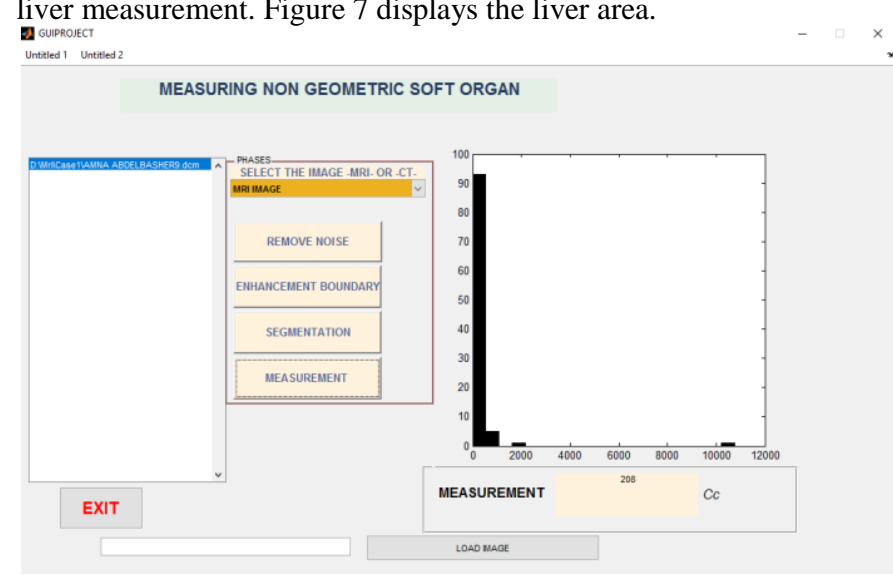

Fig7. Measurement liver volume

\section{ANALYSIS RESULT}

Regarding the results have been obtained from the prototype, where compared with Gold standard. In this paper has done ten cases, the average result displayed in table 2 compared with Gold standard manual result.

Table2. Comparison between prototype application result and Gold standard manual reading.

\begin{tabular}{|c|c|c|c|}
\hline \multirow[b]{2}{*}{ Phase } & \multicolumn{3}{|c|}{ Comparison } \\
\hline & Accuracy & $\begin{array}{l}\begin{array}{c}\text { Volume } \\
\text { error }\end{array} \\
\end{array}$ & \multirow{5}{*}{$\begin{array}{l}\text { Gold } \\
\text { standard } \\
\text { manual } \\
\text { result }\end{array}$} \\
\hline $\begin{array}{c}\text { Removing } \\
\text { noise }\end{array}$ & $99.4 \% \pm$ & $3.6 \%$ & \\
\hline Enhancement. & $98.4 \%$ & $7.2 \%$ & \\
\hline Segmentation & $98 \%$ & $\begin{array}{l}\text { CT, } 7.0 \% \\
\text { MRI } 3.6 \%\end{array}$ & \\
\hline $\begin{array}{c}\text { Measurement } \\
\text { using } \\
\text { Algorithms } \\
\text { Bank } \\
\text { template }\end{array}$ & $99.1 \%$ & $2.1 \%$ & \\
\hline
\end{tabular}

\section{CONTRIBUTION}

In this paper constructed a new method that uses algorithms bank, which uses to obtain the measurement liver volume, the algorithms bank that has been split by the phases of the prototype application. Each phase was determined algorithms according to the previous studies have proved their efficiency in this aspect, that after returning to the algorithms result. The intent of the prototype application of these four phases is to reach to the highest accuracy in measuring the liver volume compared to previous studies.

\section{REFERENCES}

[1] Am Suk Oh et al. 2014. "Design and Implementation of Standard DICOM Interface Module International Journal of Bio-Science and Bio-Technology" Vol.6, No.2 (2014), pp.141-146. 
[2] Kenji Suzuki, 2014, "Computerized Liver Volumetry on MRI

Using 3DGeodesicActiveContourSegmentation"http://www.ajronl ine.org/doi/abs/10.2214/AJR.13.10812.Jan 2015.

[3] Henderson JM, Heymsfield SB, Horowitz J, Kutner MH.1981 "Measurement of liver and spleen volume by computed tomography. Assessment of reproducibility and changes found following a selective distal splenorenal shunt. Radiology 1981; 141: 525-527 [PMID: 6974875]

[4] V.P.Ananthi, P.Balasubramaniam, C.P.Lim. 2014."Segmentation of gray scale image based on intuitionistic fuzzy sets constructed from several membership functions". PatternRecognition47(2014)38703880 .

[5] Etehad Tavakol M, Ng EYK. 2013. "Breast thermography as a potential non-contact method in the early detection of cancer: a review". J Mech Med Biol 13: 1330001; doi:10.1142/S0219519413300019.

[6] www.medicalresourcelinks.com/tac.html 2015.

[7] Gurpreet kaur , Rajdavinder Singh. 2014 Image Enhancement and It's Techniques- A Review, 2014.
International Journal of Computer Trends and Technology (IJCTT) .

[8] Mussarat Yasmin, Muhammad Sharif, Saleha Masood, Mudassar Raza and Sajjad Mohsin. 2012. Brain Image Enhancement - A Survey. World Applied Sciences Journal 17 (9): 1192-1204, 2012 ISSN 1818-4952 (C) IDOSI Publications, 2012.

[9] R. Sharmila and R. Uma.2011. "A New Approach to Image Contrast Enhancement using Weighted Threshold Histogram Equalization with Improved Switching Median Filter", International Journal of Advanced Engineering Sciences and Technologies (IJAEST), 7(2), pp. 38-43, 2011.

[10] Rafael C. Gonzalez and Richard E. Woods, "Digital Image Processing", 3rd edition, Prentice Hall, 2008.

[11] Gurpreet kaur ， Rajdavinder Singh. 2014. Image Enhancement and Its Techniques. International Journal of Computer Trends and Technology (IJCTT) - volume 12 number 3 - Jun 2014.

[12] Book. Encyclopedia of Diagnostic imaging. Albert L.Baet. Springer reference. 2015. 